\title{
Effect of rhythmic auditory cueing on gait in cerebral palsy:a systematic review and meta-analysis
}

This article was published in the following Dove Press journal:

Neuropsychiatric Disease and Treatment

\author{
Shashank Ghai' \\ Ishan Ghai \\ Alfred O. Effenberg' \\ 'Institute for Sports Science, Leibniz \\ University Hannover, Hannover, \\ Germany; ${ }^{2}$ School of Life Sciences, \\ Jacobs University, Bremen, Germany
}

Correspondence: Shashank Ghai Institut für Sportwissenschaft, Leibniz Universität, Am Moritzwinkel 6, Hannover 30167, Germany

Tel +49 511 762I 7290

Email shashank.ghai@sportwiss. uni-hannover.de

\begin{abstract}
Auditory entrainment can influence gait performance in movement disorders. The entrainment can incite neurophysiological and musculoskeletal changes to enhance motor execution. However, a consensus as to its effects based on gait in people with cerebral palsy is still warranted. A systematic review and meta-analysis were carried out to analyze the effects of rhythmic auditory cueing on spatiotemporal and kinematic parameters of gait in people with cerebral palsy. Systematic identification of published literature was performed adhering to Preferred Reporting Items for Systematic Reviews and Meta-Analyses and American Academy for Cerebral Palsy and Developmental Medicine guidelines, from inception until July 2017, on online databases: Web of Science, PEDro, EBSCO, Medline, Cochrane, Embase and ProQuest. Kinematic and spatiotemporal gait parameters were evaluated in a meta-analysis across studies. Of 547 records, nine studies involving 227 participants (108 children/119 adults) met our inclusion criteria. The qualitative review suggested beneficial effects of rhythmic auditory cueing on gait performance among all included studies. The meta-analysis revealed beneficial effects of rhythmic auditory cueing on gait dynamic index (Hedge's $g=0.9$ ), gait velocity (1.1), cadence $(0.3)$, and stride length $(0.5)$. This review for the first time suggests a converging evidence toward application of rhythmic auditory cueing to enhance gait performance and stability in people with cerebral palsy. This article details underlying neurophysiological mechanisms and use of cueing as an efficient home-based intervention. It bridges gaps in the literature, and suggests translational approaches on how rhythmic auditory cueing can be incorporated in rehabilitation approaches to enhance gait performance in people with cerebral palsy.
\end{abstract}

Keywords: entrainment, spastic diplegia, hemiplegia, ataxia, rehabilitation, balance

\section{Introduction}

Cerebral palsy is a common developmental disorder. ${ }^{1,2}$ The global prevalence of cerebral palsy is approximately $1.5-3.5 / 1,000$ children, ${ }^{3,4}$ and is supposedly growing in developing countries. ${ }^{5}$ Cerebral palsy is primarily characterized by pre/postnatal damage to the brain, ${ }^{3}$ often predisposing to grave neuromuscular and psychological disorders. ${ }^{3,6}$ The treatment of cerebral palsy inflicts substantial costs ${ }^{7}$ and adversely impacts quality of life. ${ }^{8,9}$ Typically, motor dysfunction in cerebral palsy is characterized by spastic or extrapyramidal deficits. ${ }^{10}$ These neuromuscular dysfunctions might cause dyskinesia, dystonia, ataxia, or hypotonia. ${ }^{11,12}$ Further, these might lead to increased fatigue, reduced dexterity/coordination, postural instability, muscle contracture, and joint subluxation. Also, these neuromuscular disorders progress with aging. ${ }^{11}$ For instance, lack of mobility and hypertonia often lead to development of muscle and joint contractures and secondary bone deformities. These neuromuscular deficits among both children and older adults with cerebral palsy considerably impair kinetic and kinematic changes, impair locomotion, and predispose to falls. For instance, 
exaggerated anterior stooping posture associated with increased anterior tilt in the pelvis, hip flexion, adduction, and internal rotation ${ }^{13-15}$ adversely impact efficiency in energy expenditure ${ }^{16}$ and spatiotemporal gait parameters. ${ }^{17}$ Bourgeois et $\mathrm{al}^{18}$ reported reduction in spatiotemporal gait parameters, such as cadence, stride length, and gait velocity associated with considerable enhancement in gait variability, which might predispose severely toward falls. ${ }^{19}$

In addition to these musculoskeletal changes, Rosenbaum et $\mathrm{al}^{2}$ suggested considerable discrepancies in sensory perceptions, cognition, and behavior. Neuroimaging studies report deficits in the dorsolateral prefrontal cortex, dorsal anterior cingulate gyrus, ${ }^{20}$ somatosensory cortex, ${ }^{21}$ and cerebellum ${ }^{22}$ which might considerably impair intellectual and cognitive performance. ${ }^{22-24}$ Likewise, deficits in corticospinal, thalamocortical, superior occipitofrontal and superior longitudinal pathways have also been reported. ${ }^{12,20,25}$ Together, these psychological constraints might also impair motor performance, such as in a dual-task scenario. For instance, Hung et $\mathrm{al}^{26}$ reported drops in gait-performance measures in unilateral cerebral palsy patients while performing a dual task. Studies have suggested that this modification in gait patterns might happen due to an alleviation in "internal" conscious attention toward autonomic control that adversely impacts proprioception and autonomic functioning, possibly because of movementspecific reinvestment. ${ }^{27-29}$ The theory suggests that directing attention internally to control autonomic movements, such as gait, can have an adverse impact on performance, ${ }^{29}$ especially in high-stress situations. ${ }^{30}$ Common treatment strategies to curb motor dysfunctions in cerebral palsy include training with virtual reality, ${ }^{31}$ biocueing, ${ }^{32}$ physical/occupational therapy, ${ }^{33}$ physical exercise, ${ }^{34}$ treadmill, ${ }^{35}$ and orthosis. ${ }^{36,37}$

Recently, several studies have tried to address the sensorimotor deficits in people with cerebral palsy by applying rhythmic auditory entrainment. ${ }^{38-41}$ Cueing aims to counteract sensory deficits, and has been shown to modulate neuromagnetic $\beta$-oscillations, ${ }^{42}$ cortical reorganization, enhance biological motion perception, ${ }^{43,44}$ motor imagery, ${ }^{45,46}$ neural plasticity, ${ }^{48}$ reduce shape variability in musculoskeletalactivation patterns, ${ }^{47}$ and movement-specific reinvestment. ${ }^{49}$ Moreover, as a cheap ${ }^{50}$ and viable ${ }^{51}$ treatment strategy, this approach can provide substantial benefits in developing countries, where prevalence of cerebral palsy due to socioeconomic factors is more prominent. ${ }^{52,53}$ We identified highquality systematic reviews analyzing the effects of external auditory cueing on gait performance among healthy, ${ }^{121}$ Parkinsonism ${ }^{54-56,122}$ and stroke participants. ${ }^{57-59}$ However, to the best of our knowledge, no systematic or narrative analysis has been carried out to analyze the effects of auditory entrainment on gait in people with cerebral palsy. Therefore, we attempted to develop a state of knowledge for the use of cerebral palsy patients and medical practitioners, where both qualitative and quantitative data from high-quality studies can be interpreted.

\section{Materials and methods}

This review was conducted according to the guidelines outlined in the PRISMA (Preferred Reporting Items for Systematic Reviews and Meta-Analyses) statement ${ }^{60}$ and American Academy for Cerebral Palsy and Developmental Medicine (AACPDM) methodology for systematic reviews. ${ }^{61}$

\section{Data sources and search strategy}

The academic databases Web of Science, PEDro, EBSCO, Medline, Cochrane Central Register of Controlled Trials, Embase and ProQuest were searched from inception until July 2017. A sample-search strategy is provided in Table 1.

\section{Data extraction}

Upon selection for review, data extracted from each article were study aim, selection criteria, sample size, sample description (sex, age, health status), intervention, characteristics of auditory cueing, dual tasks, outcome measures, results, and conclusions. The data were then summarized and tabulated (Table 2). The inclusion criteria for the studies were: randomized controlled trials, cluster-randomized controlled trials, or controlled clinical trials; reporting reliable and valid spatiotemporal gait and kinematic parameters; including dynamic aspects of gait stability; use of PEDro methodological quality scale (score $\geq 4$ ); conducted on human participants; published in a peer-reviewed academic journal; and published in English, German, or Korean.

\section{Quality and risk-of-bias assessment}

The quality of the studies was assessed using the PEDro methodological quality scale. ${ }^{62}$ The scale consists of eleven items addressing external validity, internal validity, and interpretability, and can detect potential bias with high reliability, ${ }^{63}$ and validity. ${ }^{62} \mathrm{~A}$ blinded rating of the methodological quality of the studies was carried out by the first (SG), second (IG) and third (AOE) reviewers. Ambiguous issues were discussed between reviewers and consensus was reached. Included studies were rated and interpreted according to scoring of 9-10, 6-8, and 4-5 for "excellent", "good", and "fair" quality, ${ }^{64}$ respectively. Inadequate randomization, 
Table I Sample search strategy - Embase

\begin{tabular}{|c|c|}
\hline Date & July 10, 2017 \\
\hline$\overline{\text { Strategy }}$ & \#I AND \#2 AND \#3 AND \#4 AND \#5 AND \#6 AND \#7 \\
\hline$\# I$ & 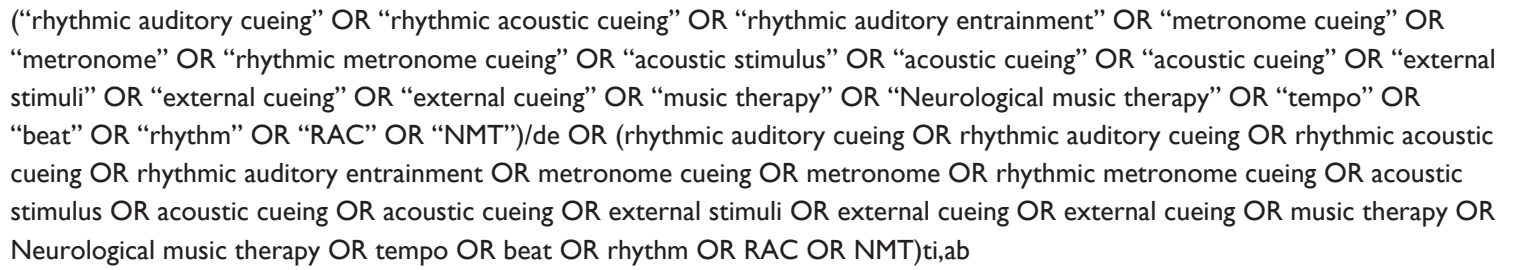 \\
\hline$\# 2$ & 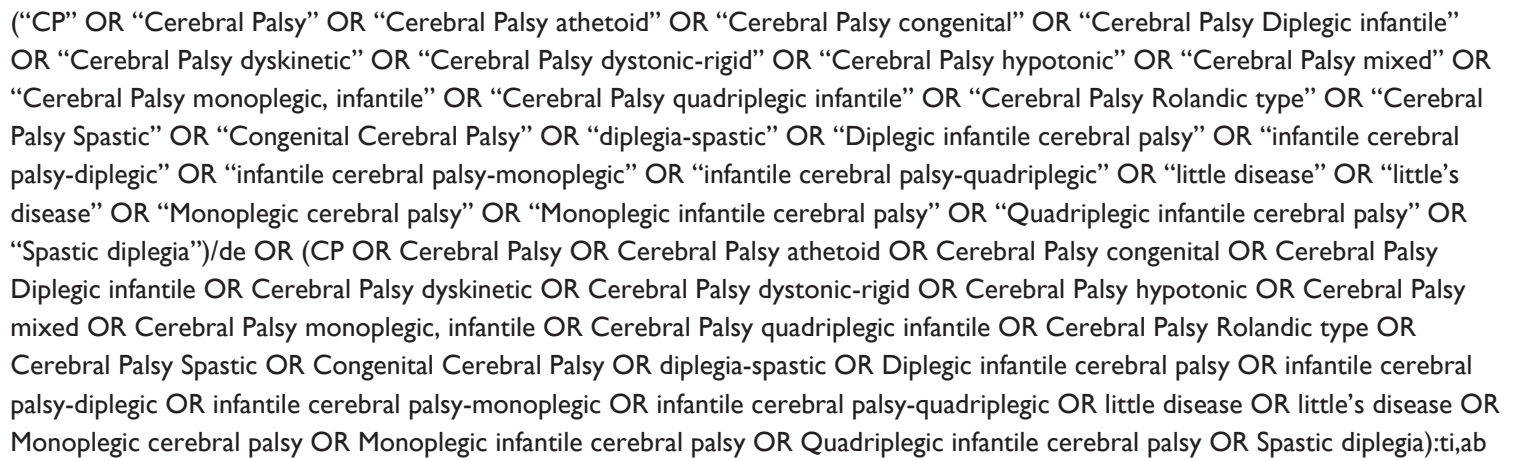 \\
\hline \#3 & $\begin{array}{l}\text { (“cognitive task" OR “concurrent task” OR “dual task" OR “dual task paradigm” OR “dual task paradigm” OR “cognitive task } \\
\text { training” OR "dual task training” OR "dual task training")/de OR (cognitive task OR concurrent task OR dual task OR dual task OR } \\
\text { dual task paradigm OR dual task paradigm OR cognitive task training OR dual task training OR dual task training”):ti,ab }\end{array}$ \\
\hline$\# 4$ & $\begin{array}{l}\text { ("walking" OR "gait" OR "locomotion" OR "range of motion" OR "ROM" OR "ambulation" OR "mobility" OR "treadmill gait" } \\
\text { OR "balance" OR "stability" OR "stride" OR "gait training" OR "gait rehabilitation")/de OR (walking OR gait OR locomotion OR } \\
\text { range of motion OR ROM OR ambulation OR mobility OR treadmill gait OR balance OR stability OR stride OR gait training OR gait } \\
\text { rehabilitation);ti,ab }\end{array}$ \\
\hline$\# 5$ & $\begin{array}{l}\text { ("rehabilitation" OR "treatment" OR "rehab" OR "management" OR "therapy" OR “physiotherapy" OR "physical therapy" OR } \\
\text { "prevention" OR "risk prevention")/de OR (rehabilitation OR treatment OR rehab OR management OR therapy OR physiotherapy } \\
\text { OR physical therapy OR prevention OR risk prevention);tiab }\end{array}$ \\
\hline \#6 & $\begin{array}{l}\text { ("age groups" OR "adolescent" OR "young" OR "elderly" OR "old” AND ("gender" OR "male" OR "female")/de OR (age groups } \\
\text { OR adolescent OR young OR elderly OR old AND (gender OR male OR female));ti;ab }\end{array}$ \\
\hline \#7 & $\begin{array}{l}\text { ("intervention study" OR "cohort analysis" OR "longitudinal study" OR "cluster analysis" OR "crossover trial" OR "cluster analysis" } \\
\text { OR "randomized trial” OR "major clinical study")/de OR (longitudinal OR cohort OR crossover trial OR cluster analysis OR } \\
\text { randomized trial OR clinical trial OR controlled trial);ti,ab }\end{array}$ \\
\hline
\end{tabular}

nonblinding of assessors, no intention-to-treat analysis, and no measurement of compliance were considered as major threats for biasing. ${ }^{65}$

\section{Data analysis}

This systematic review also included a meta-analysis approach to develop a better understanding of the incorporated interventions. ${ }^{66}$ Presence and lack of heterogeneity drove the use of either random- or fixed-effect meta-analysis. ${ }^{67}$ A narrative synthesis of the findings structured according to intervention, population characteristics, methodological quality, and type of outcome is provided (Table 2). A meta-analysis was conducted between pooled studies using Comprehensive Meta-Analysis software (version 2.0; Biostat, Englewood, NJ, USA). Heterogeneity among the studies was assessed using $I^{2}$ statistics. The data in this review were systematically distributed, and for each available variable pooled, dichotomous data were analyzed and forest plots with 95\% CIs reported. Effect sizes were adjusted and reported as Hedge's $g .{ }^{68}$ Thresholds for interpretation of effect sizes were as follows: standard mean effect of 0 meant no change, negative effect meant a negative change, and a positive effect meant a positive change.

Mean effect of 0.2 was interpreted as a small effect, 0.5 a medium effect, and 0.8 a large effect. ${ }^{69}$ Interpretation of heterogeneity via $I^{2}$ statistics was $25 \%, 50 \%, 75 \%$ as negligible, moderate and substantial heterogeneity, respectively. $75 \%$ as negligible, moderate, and substantial heterogeneity, respectively. Meta-analysis reports, including heterogeneity among studies, were evaluated to determine the reason for heterogeneity, and studies included were then pooled separately and analyzed again in a sub-group analysis. The $\alpha$-level was set at $95 \%$. 


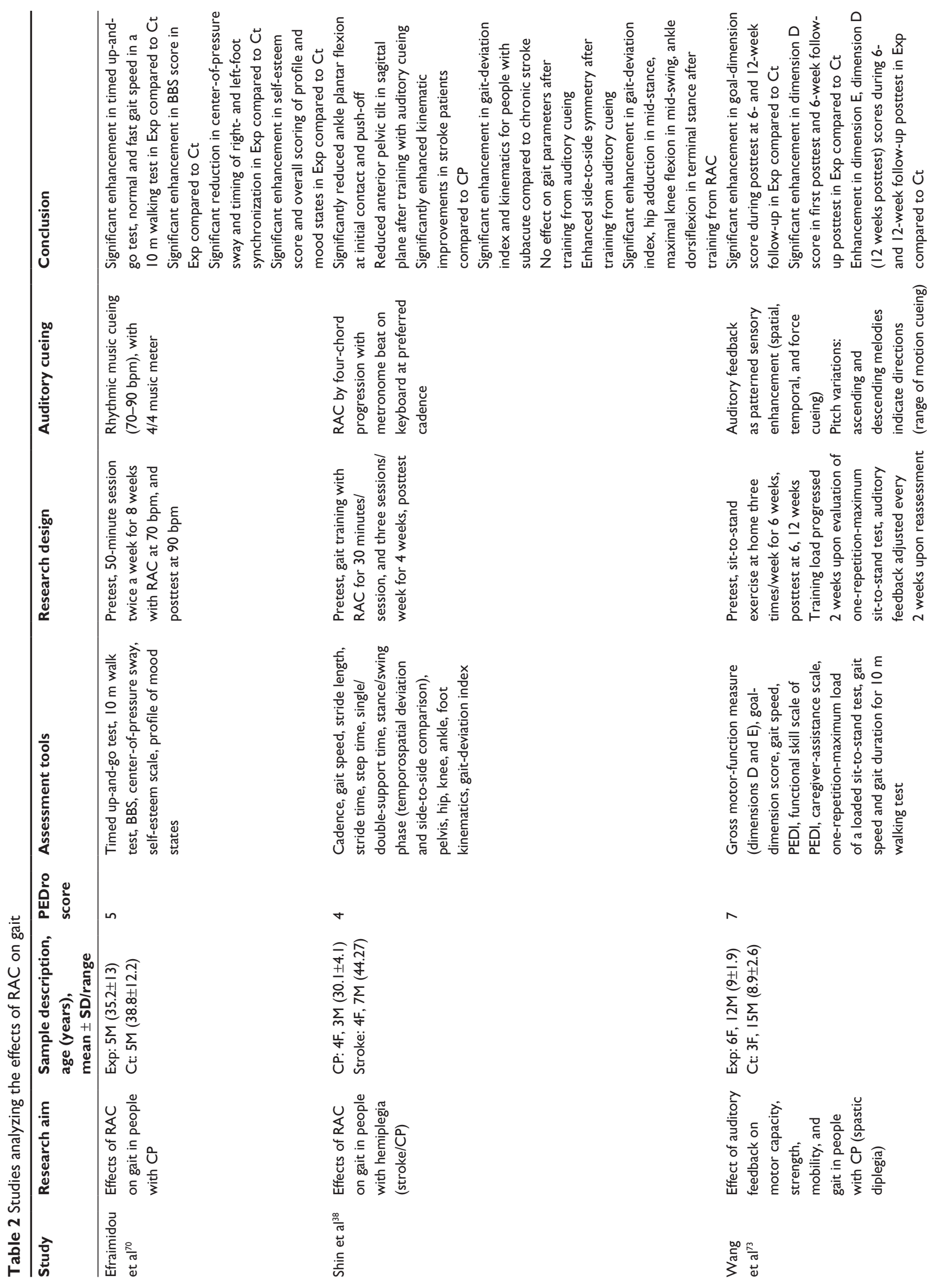



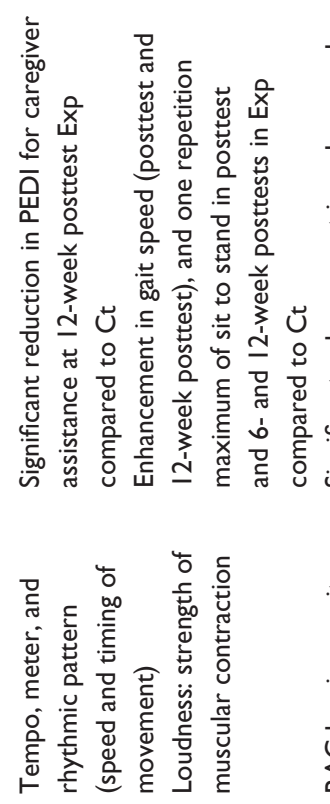
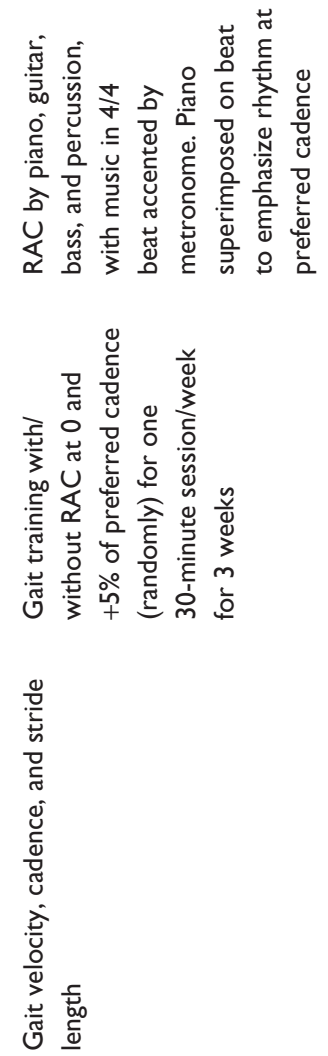

$\Lambda$

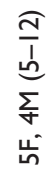

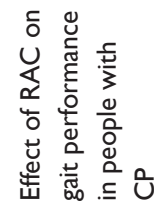

要

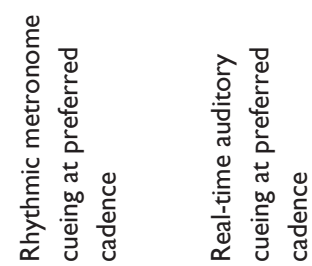

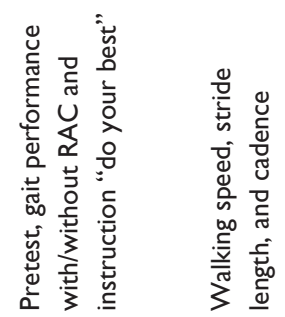

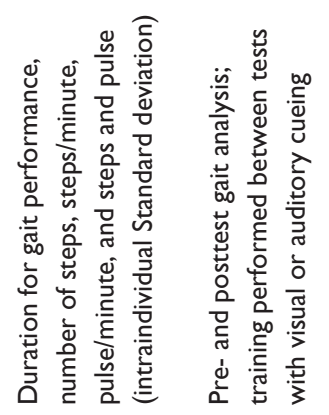

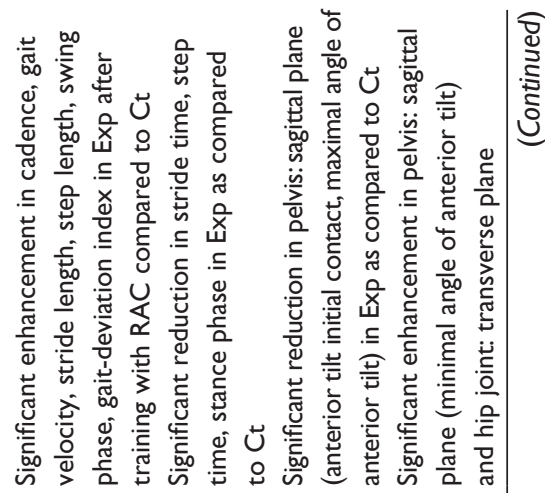

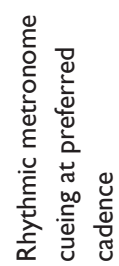
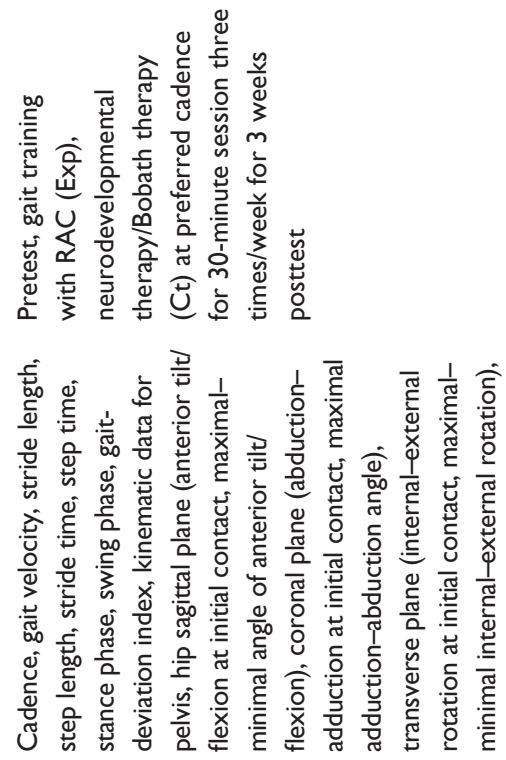

$\wedge$

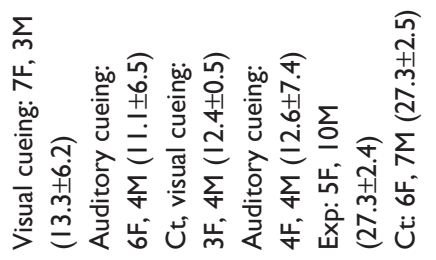

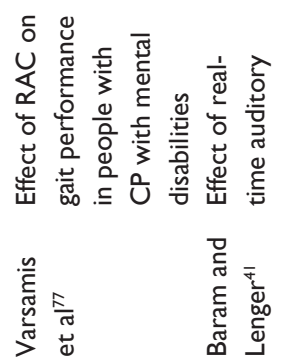

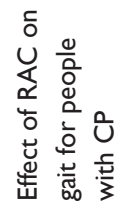

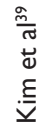




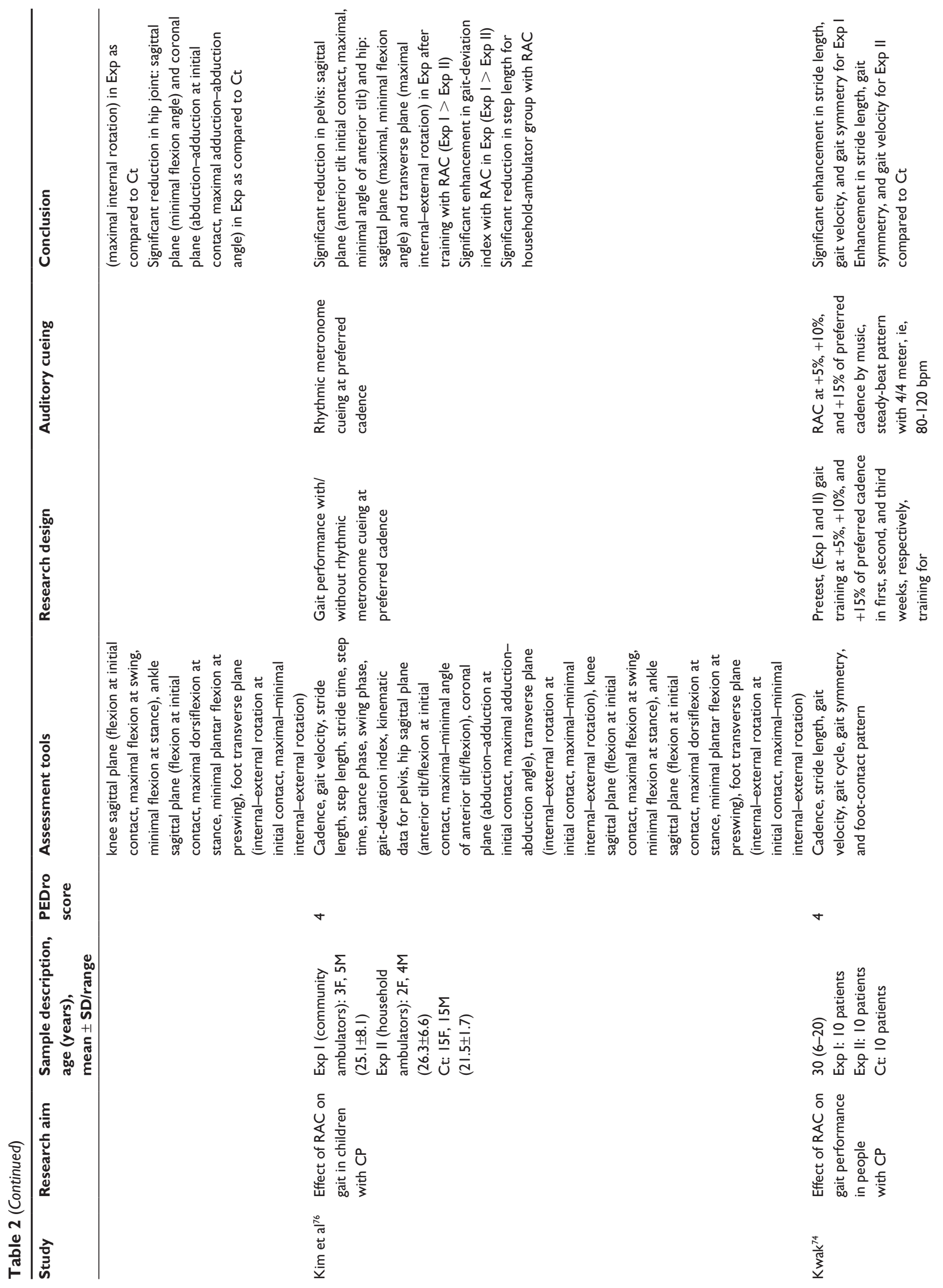




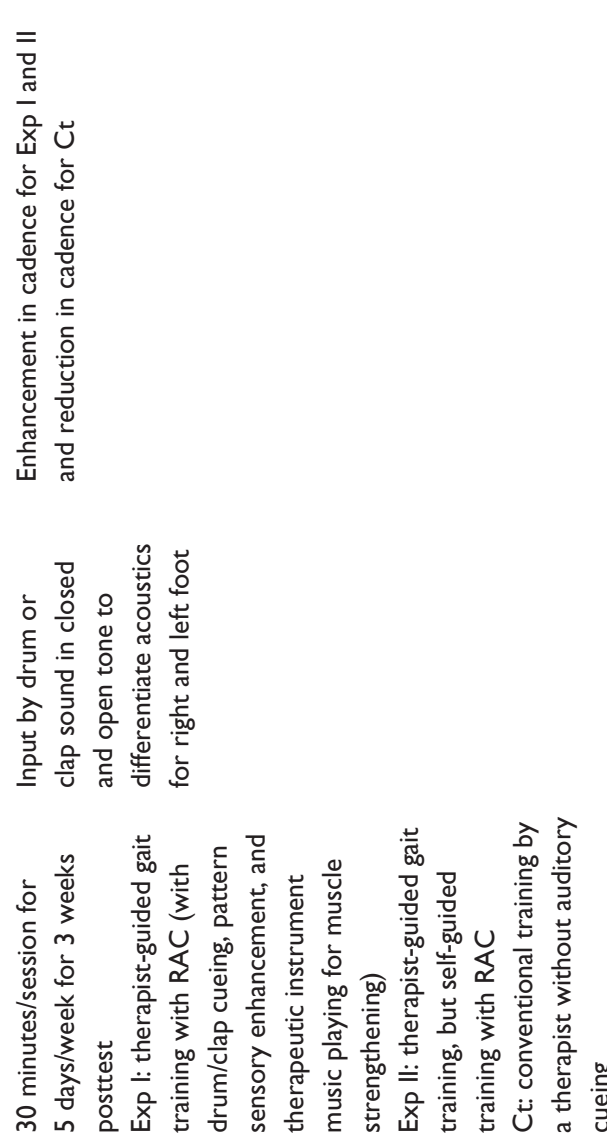

\section{Results}

\section{Characteristics of studies included}

Our initial search across academic databases yielded a total of 387 studies; 175 studies were included from a personal library. After implementing our inclusion/exclusion criteria, nine studies were left (Figure 1). Data from the studies included have been summarized in Table 2. Of the nine studies included, all were controlled clinical trials.

\section{Participants}

A total of 227 participants were analyzed in the incorporated studies. In the studies included, eight studies incorporated mixed-sex patients. Only one study included male participants. ${ }^{70}$ The studies provided data on 227 participants ( $\mathrm{n}=119$ females/108 males). Moreover, in 108 children, the sex distribution was 57 females to 51 males, and for adults 62 females to 57 males. Descriptive statistics relating to the age (means $\pm \mathrm{SD}$ ) of the participants were tabulated across the studies (Table 2).

\section{Risk of bias}

To reduce the risks of bias, studies scoring $\geq 4$ on PEDro were included in the review. Moreover, research protocols to be included in the review were limited to gold-standard randomized controlled trials, cluster-randomized controlled trials, and controlled clinical trials. The individual scores attained by studies using the PEDro scale are reported in Table 2, and Table S1. The average PEDro score for the nine studies was 5 of 10 , indicating fair quality for studies overall. Three studies scored 7, and six studies scored 4 . Risk of bias across the studies is shown in Figure 2.

\section{Outcomes}

The results provided evidence for a positive impact of rhythmic auditory cueing on spatiotemporal and kinematic gait parameters among adults and children with cerebral palsy. In all studies, significant enhancement in primary spatiotemporal and kinematic gait parameters were reported.

\section{Meta-analyses}

The evaluation of research studies via meta-analysis requires strict inclusion criteria to limit heterogeneity efficiently. ${ }^{71}$ However, among the pooled group of studies after strict inclusion criteria, some unexplained heterogeneity was still observed. Subgroup analyses were then performed for identical studies to evaluate the cause of the heterogeneity. The parameters evaluated were spatiotemporal gait parameters, such as cadence, stride length, gait velocity, and kinematic 


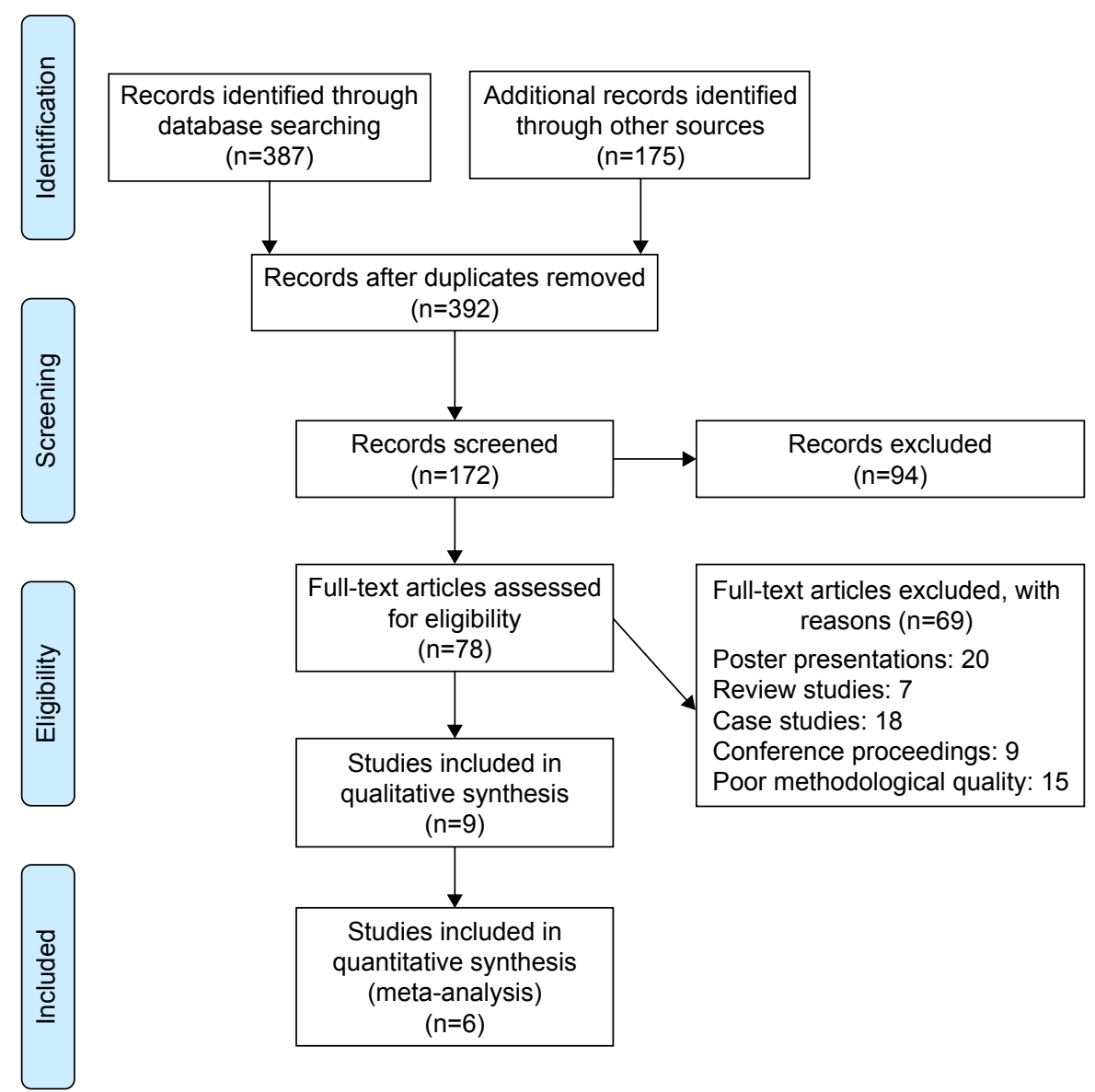

Figure I PRISMA (Preferred Reporting Items for Systematic Reviews and Meta-Analyses) flowchart for inclusion of studies.

parameters. Further analyses were conducted to evaluate the effects of rhythmic auditory cueing at preferred cadence on gait velocity in both adults and children separately. We included a generalized group analysis by first combining all the pooled studies. The studies excluded differed considerably in assessment methods or if descriptive statistics were not mentioned in the manuscript. However, attempts were made to contact the coauthors for the data.

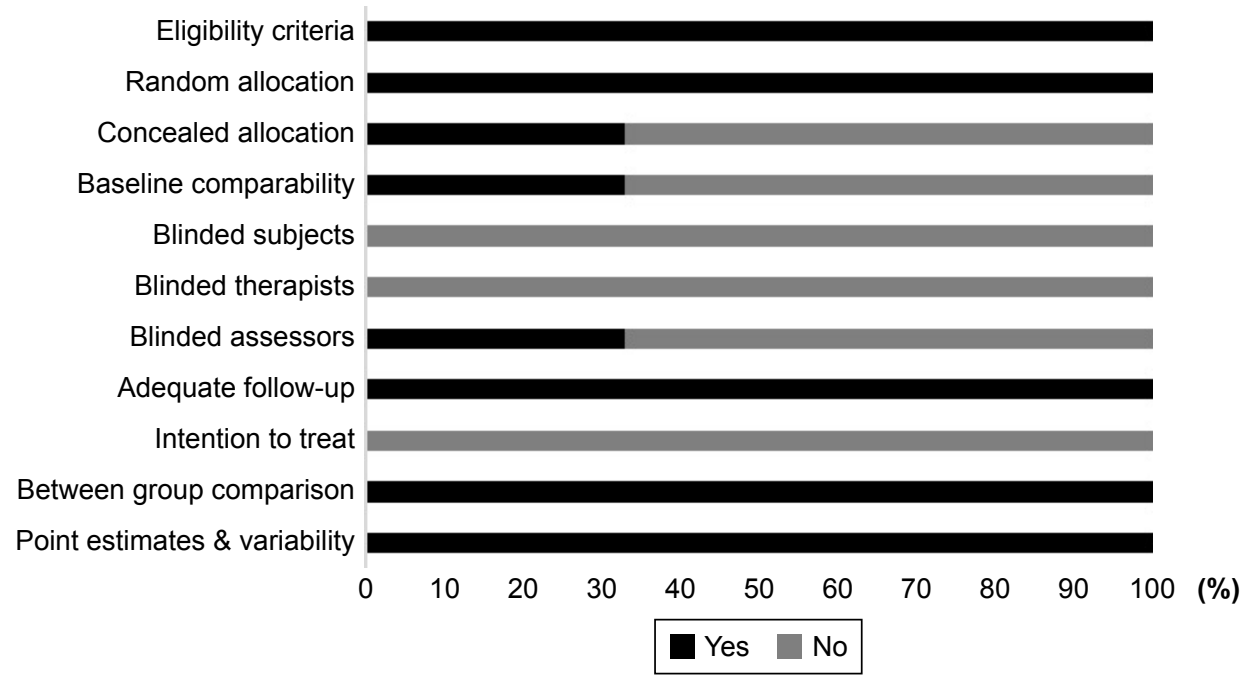

Figure 2 Risk of bias across studies. 


\section{Gait velocity}

Gait velocity was analyzed in six studies. Here, two studies evaluated the effects of rhythmic auditory cueing on gait velocity in adults ${ }^{38,72}$ and four in children ${ }^{41,73-75}$ with cerebral palsy. One study included assessment of gait velocity while using patterned sensory enhancement ${ }^{73}$ as the mode of auditory feedback. Analysis of studies revealed (Figure 3) a large positive effect ( $g=1.13,95 \%$ CI $0.33-1.94)$. Substantial heterogeneity was observed between studies $\left(I^{2}=84 \%\right.$, $P>0.01)$. Subgroup analyses were conducted to explore heterogeneity.

An analysis for effects of rhythmic auditory cueing on gait velocity in children revealed (Figure S1), large positive effect with substantial heterogeneity $(g=1.24,95 \%$ CI $0.31-2.17$, $\left.I^{2}=81 \% ; P<0.01\right)$. Here, the heterogeneity could possibly be attributed to different training regimes in the studies, ie, no training was included by one, ${ }^{41}$ while others ${ }^{73-75}$ had training regimes for $\geq 3$ weeks. Subgroup analysis revealed (Figure S2) a large positive effect with substantial heterogeneity $(g=1.53,95 \%$ CI $1.07-1.98, P=82 \% ; P<0.01)$. Moreover, Jiang ${ }^{75}$ included only one training session per week. Whereas, others performed training for three (Wang et al. ${ }^{73}$ ), and five times $\left(\mathrm{Kwak}^{74}\right)$, per week. Subgroup analysis revealed a large positive effect with negligible heterogeneity $(g=2.05,95 \% \mathrm{CI}$ $\left.1.5-2.6, I^{2}=0 ; P>0.05\right)$. Finally, subgroup analysis evaluating the effects of rhythmic auditory cueing on gait velocity in adults revealed a large positive effect with negligible heterogeneity $\left(g=0.95,95 \% \mathrm{CI}-0.95\right.$ to $\left.2.85, I^{2}=0 ; P>0.05\right)$.

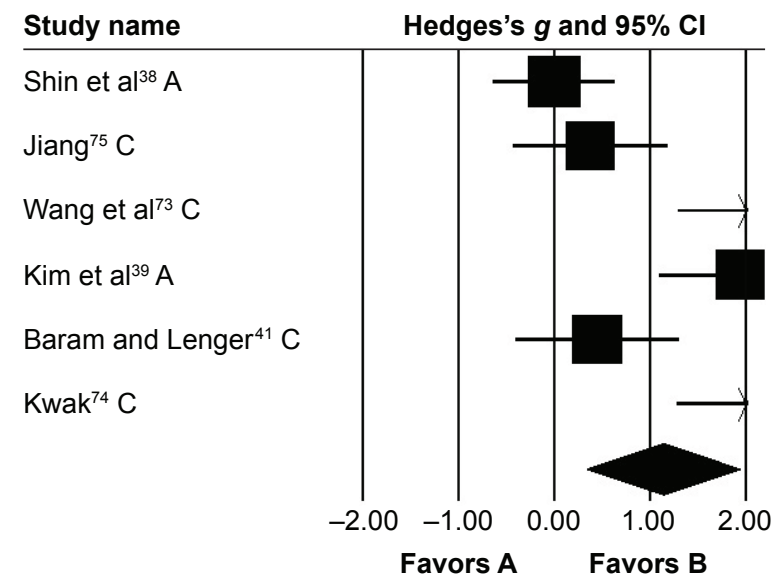

Figure 3 Forest plot illustrating individual studies evaluating the effects of rhythmic auditory cueing on gait velocity in people with cerebral palsy.

Notes: Negative effects indicate reduction in gait velocity, positive effects enhancement in gait velocity. Weighted-effect sizes - Hedge's g (boxes) and $95 \% \mathrm{Cl}$ (whiskers) - demonstrate repositioning errors for individual studies. The diamond represents pooled effect sizes and $95 \% \mathrm{Cl}$. Negative mean differences indicate favorable outcomes for control groups, positive mean differences favorable outcomes for experimental groups.

Abbreviations: $\mathrm{A}$, adults; $\mathrm{C}$, children.

\section{Stride length}

Stride length was analyzed in five studies. Two and three studies evaluated the effects of rhythmic auditory cueing on stride length in adults ${ }^{38,72}$ and children, ${ }^{41,73-75}$ respectively. Analysis revealed (Figure 4) a medium positive effect ( $g=0.58,95 \%$ CI -0.02 to 1.19 ). Moderate heterogeneity was observed between studies $\left(I^{2}=65 \%, P>0.01\right)$. Subgroup analyses were conducted to explore the cause of heterogeneity. Analysis for effects of rhythmic auditory cueing on stride length in children revealed (Figure S3) a medium positive effect with negligible heterogeneity ( $g=0.75,95 \%$ CI $\left.0.01-1.48, I^{2}=0 ; P>0.05\right)$. Subgroup analysis evaluating the effects of rhythmic auditory cueing on stride length in adults revealed a comparably smaller medium effect with negligible heterogeneity $(g=0.3,95 \% \mathrm{CI}-1.07$ to 1.67 , $\left.I^{2}=0 ; P>0.05\right)$.

\section{Cadence}

Cadence was analyzed in five studies, of which two evaluated the effects of rhythmic auditory cueing on cadence in adults $^{38,72}$ and three in children ${ }^{41,73-75}$ with cerebral palsy. Analysis of studies revealed (Figure 5) a medium positive effect ( $g=0.33,95 \%$ CI -0.41 to 1.07 ). Substantial heterogeneity was observed between studies $\left(I^{2}=79 \%, P>0.01\right)$. Subgroup analyses were conducted to explore heterogeneity. An analysis for effects of rhythmic auditory cueing on cadence in children revealed a small negative effect with negligible heterogeneity ( $g=-0.11,95 \% \mathrm{CI}-0.97$ to 0.74 , $\left.I^{2}=0 ; P>0.05\right)$. Subgroup analysis evaluating the effects of

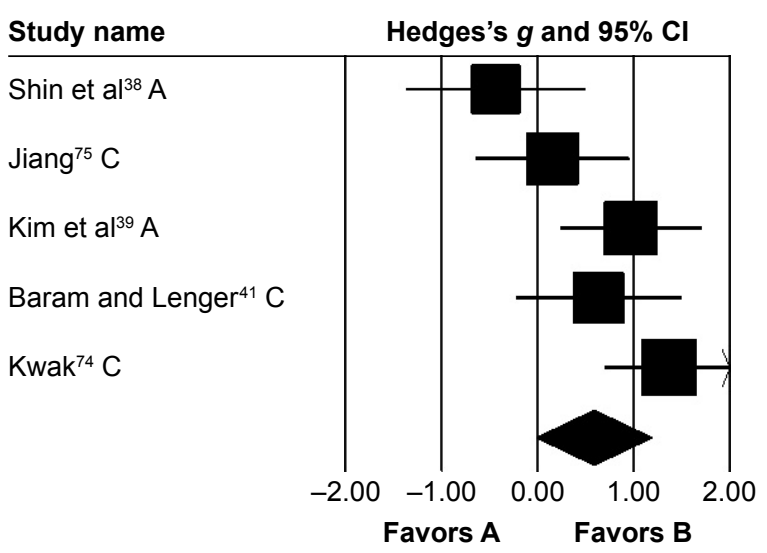

Figure 4 Forest plot illustrating individual studies evaluating the effects of rhythmic auditory cueing on stride length in people with cerebral palsy.

Notes: Negative effects indicate reduction in stride length, positive effects enhancement in stride length. Weighted-effect sizes - Hedge's g (boxes) and $95 \% \mathrm{Cl}$ (whiskers) - demonstrate repositioning errors for individual studies. The diamond represents pooled effect sizes and $95 \% \mathrm{Cl}$. Negative mean differences indicate favorable outcomes for control groups, positive mean differences favorable outcomes for experimental groups.

Abbreviations: $\mathrm{A}$, adults; $\mathrm{C}$, children. 


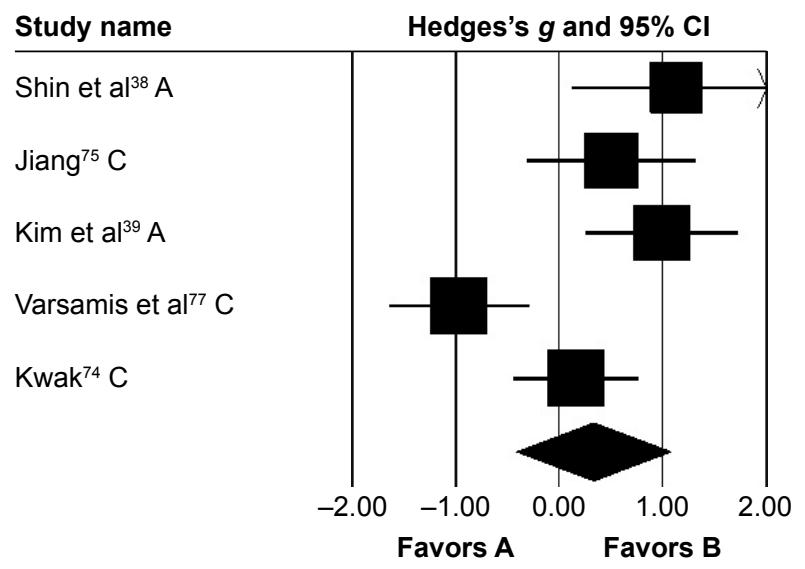

Figure 5 Forest plot illustrating individual studies evaluating the effects of rhythmic auditory cueing on cadence in people with cerebral palsy.

Notes: Negative effects indicate reduction in step frequency, positive effects enhancement in step frequency. Weighted-effect sizes - Hedge's g (boxes) and $95 \% \mathrm{Cl}$ (whiskers) - demonstrate repositioning errors for individual studies. The diamond represents pooled effect sizes and $95 \% \mathrm{Cl}$. Negative mean differences indicate favorable outcomes for control groups, positive mean differences favorable outcomes for experimental groups.

Abbreviations: $\mathrm{A}$, adults; $\mathrm{C}$, children.

rhythmic auditory cueing on cadence in adults revealed a large positive effect with negligible heterogeneity $(g=1.04$, 95\% CI 0.44-1.64, $\left.I^{2}=0 ; P>0.05\right)$.

\section{Kinematic parameters}

Three studies analyzed the effects of rhythmic auditory cueing on gait-dynamic index (a combined measure of lowerlimb kinematic performance). Data for subgroup analysis on the gait dynamic index concerning community and household dwellers were extracted from two studies. ${ }^{38,76}$ Analysis revealed (Figure 6) a large positive effect $(g=0.92,95 \%$ CI

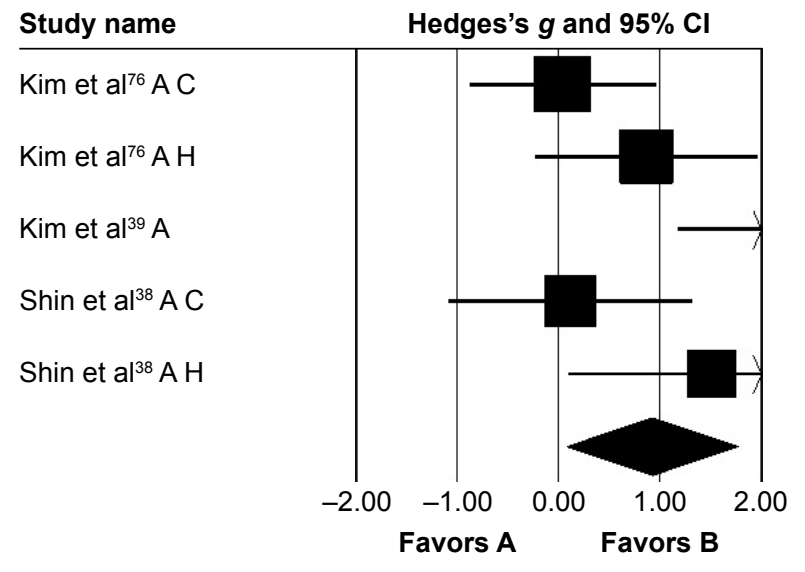

Figure 6 Forest plot illustrating individual studies evaluating the effects of rhythmic auditory cueing on gait-dynamic index in people with cerebral palsy.

Notes: Negative effects indicate reduction in gait-dynamic index, positive effects enhancement in gait-dynamic index. Weighted effect sizes - Hedge's $g$ (boxes) and $95 \% \mathrm{Cl}$ (whiskers) - demonstrate repositioning errors for individual studies. The diamond represents pooled effect sizes and $95 \% \mathrm{Cl}$. Negative mean differences indicate a favorable outcome for control groups, positive mean differences a favorable outcome for experimental groups.

Abbreviations: A, adults; $\mathrm{C}$, children; $\mathrm{H}$, household dwellers. $\left.0.07-1.76, I^{2}=0 ; P<0.01\right)$ with negligible heterogeneity. Further, an analysis of gait-dynamic index in community dwellers revealed a small positive effect with negligible heterogeneity ( $g=0.07,95 \% \mathrm{CI}-0.66$ to $0.8, I^{2}=0 ; P>0.05$ ). Comparably, analysis of household dwellers revealed a large positive effect with negligible heterogeneity $(g=1.11,95 \%$ CI $\left.0.24-1.98, I^{2}=0 ; P>0.05\right)$. Subgroup analysis was also conducted on individual kinematic parameters to specify the magnitude of effects of rhythmic auditory cueing on specific joint kinematics.

Subgroup analysis evaluating changes at the pelvis revealed (Figure S4) small negative effects with negligible heterogeneity ( $g=-0.23,95 \% \mathrm{CI}-0.68$ to $0.21, I^{2}=0$; $P>0.05)$. At the hip joint, medium negative effects with moderate heterogeneity ( $g=-0.43,95 \% \mathrm{CI}-0.89$ to 0.01 , $I^{2}=33.5 \% ; P>0.01$ ) were observed (Figure S5). At the knee joint, medium positive effects with negligible heterogeneity ( $g=0.26,95 \% \mathrm{CI}-0.18$ to $0.71, I^{2}=0 ; P>0.05$ ) were observed (Figure $\mathrm{S} 6$ ). At the ankle joint, medium positive effects with moderate heterogeneity $(g=0.36,95 \% \mathrm{CI}-0.09$ to 0.81 , $I^{2}=32.7 \% ; P>0.01$ ) were observed (Figure S7). Finally, at the foot, small negative effects with moderate heterogeneity ( $g=-0.18,95 \% \mathrm{CI}-0.62$ to $0.26, I^{2}=0 ; P>0.05$ ) were observed (Figure S8).

\section{Discussion}

The primary objective of this systematic review and metaanalysis was to synthesize the current state of knowledge for the effects of rhythmic auditory cueing on gait in people with cerebral palsy. All nine studies reported beneficial effects of rhythmic auditory cueing on gait parameters in children and adults with cerebral palsy. Further, the meta-analysis found significant small-large standardized effects for the benefits of rhythmic auditory cueing on spatiotemporal and kinematic parameters of gait among patients affected with cerebral palsy.

Typically, spatiotemporal parameters of gait may worsen over time in those with cerebral palsy. Deficits in periventricular white matter, ${ }^{12}$ gray matter, ${ }^{78}$ cerebellum, ${ }^{79}$ basal ganglia, ${ }^{80}$ and thalamus ${ }^{81}$ have been well documented. ${ }^{12}$ These neural centers play an integral role in managing stabilization and performance during automated tasks, such as posture and gait. ${ }^{82,83}$ In addition, increasing psychological stress might be exerted on automated control for posture, gait, and cognitive processing by deficits reported in corticospinal, thalamocortical, superior occipitofrontal, and longitudinal pathways,${ }^{84-86}$ possibly also explaining the loss of gait rhythmicity. ${ }^{87}$ Likewise, increased energy expenditure, ${ }^{88}$ associated variability in muscle contraction, and force production 
add to the instability. ${ }^{89}$ Rhythmic auditory cueing seems to counter these deficits efficiently. The current meta-analysis reported enhancements in gait velocity (1.24) and stride length $(0.75)$ in children and gait velocity $(0.95)$, stride length (0.3), and cadence (1.04) in adults. Beneficial effects were also observed in gross gait-dynamic index (a combined measure of kinematic variables during gait) for adult patients affected with cerebral palsy (0.92).

Several mechanisms have been suggested for the beneficial effects of rhythmic auditory cueing. For instance, auditory entrainment might aid in reducing errors while executing gait by guiding specific movement patterns ${ }^{90,91}$ External entrainment might act as guidance for "heel-contact" and "push-off" timing and/or muscle contractions..$^{39}$ Likewise, such crosssensory cueing might also reduce information overload in the native sensory modality by directing task-irrelevant information toward the underused sensory modality. ${ }^{92}$ The application of auditory entrainment is believed to allow enhancement in gait performance by bypassing or facilitating the frontostriatal pathway via alternative pathways. ${ }^{93-95}$ Cunnington et $\mathrm{al}^{96}$ reaffirmed and suggested that rhythmic cueing might directly serve as an input supplementary motor area, thereby reducing the onset of motor deficits and aiding in performance. Moreover, cueing has been shown to allow modulation of neuromagnetic $\beta$-oscillations in the auditory cortex, cerebellum, inferior frontal gyrus, somatosensory area, and sensorimotor cortex ${ }^{42}$ and reduce hemispheric asymmetry ${ }^{97}$ Also, enhanced activation in inferior colliculi, ${ }^{98}$ cerebellum, brain stem, ${ }^{94,99}$ and sensorimotor cortex ${ }^{100,101}$ have been reported. This might also suggest the facilitation of corticocerebellar network reorganization. ${ }^{48}$ Finally, entrainment has also been shown to reduce variability in electromyographic activity ${ }^{102}$ and optimize velocity/acceleration profiles of joint motions by scaling movement time, ${ }^{103}$ thereby allowing stable pattern generation.

Studies have shown that rhythmic auditory cueing might also be an efficient tool to counteract dual-task-associated information-processing constraints. ${ }^{121,122}$ For instance, Lohnes and Earhart ${ }^{104}$ suggested that rhythmic entrainment might allow alleviation in gait performance by possibly freeing up cognitive resources for dual-task execution. Although dualtask performance has been shown to reduce performance in people with cerebral palsy, ${ }^{26}$ we did not identify any study analyzing the effects of rhythmic auditory cueing under higher information-processing constraints. We suggest future studies address this substantial gap in the literature. Moreover, recent studies evaluating the effects of rhythmic entrainment have revealed beneficial effects of action-relevant acoustic input on gait performance ${ }^{105,122,123}$ as compared to normal isosynchronous cueing. ${ }^{106}$ Ecologically valid action-related sounds have been suggested to enhance salience of sensory information concerning spatiotemporal information, thereby aiding movement execution. ${ }^{105-107}$ Moreover, recent research has revealed the possibilities of including emotional, ${ }^{108}$ motivational, ${ }^{109}$ and expressiveness ${ }^{110}$ components in auditory entrainment to portray differential effects on gait parameters. Unfortunately, a lack of pertinent literature concerning the specific type of modified auditory cueing in cerebral palsy limits our interpretation of the type of auditory cueing that might be beneficial in rehabilitation. Therefore, we suggest future studies address this gap.

Finally, we believe that auditory entrainment might be efficient because of its economical nature and high viability. ${ }^{50,51}$ The rhythmic entrainment factor could be utilized with music in rehabilitation and day-to-day lives. This could allow benefits in psychophysiological domains. ${ }^{111,112}$ Moreover, it is important to consider that the retention of enhancements in gait parameters relies not only on the training received in the clinic but also largely on how much the patient follows the treatment protocol at home. In the present meta-analyses, enhancements in kinematic gait parameters observed for household ambulators (1.11) were considerably larger compared to community ambulators (0.07). We believe that delivering this type of home-based intervention could be beneficial for people lacking access to medical interventions in developing countries. ${ }^{113}$ The growing number of smartphone devices in developing countries ${ }^{114}$ can be used as a delivery tool while using a simple metronome app, such as WalkMate ${ }^{115}$ or ListenMee, ${ }^{116}$ which with proper medical guidance might allow curbing of motor deficits associated with aging. ${ }^{117} \mathrm{We}$ also suggest the use of rhythmic auditory cueing as an adjunct to other rehabilitation strategies, eg, assistive devices, ${ }^{16,124,125}$ swimming, or other aquatic exercise regimes, ${ }^{118}$ as it might enhance stabilityassociated quality of life $\mathrm{e}^{119,120}$ and rehabilitation progress by focusing on psychophysiological components.

In conclusion, to the best of our knowledge, this review analyzes for the first time the effects of auditory entrainment on adults and children with cerebral palsy. The present findings are in agreement with systematic reviews and meta-analyses carried out to analyze auditory entrainment effects on healthy, ${ }^{121}$ stroke $^{57}$ and parkinsonism population groups. ${ }^{54,56,122}$ This review suggests the incorporation of rhythmic auditory cueing for enhancing gait performance and stability in people with cerebral palsy.

\section{Acknowledgment}

Publication of this article was funded by the Open Access Fund of Leibniz Universität, Hannover. 


\section{Author contributions}

SG conceptualized the study, carried out the systematic review and statistical analysis, and wrote the paper. IG and AOE were involved in the systematic review process and reviewed the final manuscript. All authors contributed toward data analysis, drafting and revising the paper and agree to be accountable for all aspects of the work.

\section{Disclosure}

The authors report no conflicts of interest in this work.

\section{References}

1. Kuperminc MN, Stevenson RD. Growth and nutrition disorders in children with cerebral palsy. Dev Disabil Res Rev. 2008;14(2): 137-146.

2. Rosenbaum P, Paneth N, Leviton A, et al. A report: the definition and classification of cerebral palsy April 2006. Dev Med Child Neurol Suppl. 2007;109:8-14.

3. Eunson P. Aetiology and epidemiology of cerebral palsy. Paediatr Child Health. 2012;22(9):361-366.

4. Yeargin-Allsopp M, Braun KV, Doernberg NS, Benedict RE, Kirby RS, Durkin MS. Prevalence of cerebral palsy in 8-year-old children in three areas of the United States in 2002: a multisite collaboration. Pediatrics. 2008;121(3):547-554.

5. Gladstone M. A review of the incidence and prevalence, types and aetiology of childhood cerebral palsy in resource-poor settings. Ann Trop Paediatr. 2010;30(3):181-196.

6. Aisen ML, Kerkovich D, Mast J, et al. Cerebral palsy: clinical care and neurological rehabilitation. Lancet Neurol. 2011;10(9):844-852.

7. Kruse M, Michelsen SI, Flachs EM, Brønnum-Hansen H, Madsen M, Uldall P. Lifetime costs of cerebral palsy. Dev Med Child Neurol. 2009; 51(8):622-628.

8. Vargus-Adams J. Health-related quality of life in childhood cerebral palsy. Arch Phys Med Rehabil. 2005;86(5):940-945.

9. Mohammed FM, Ali SM, Mustafa MA. Quality of life of cerebral palsy patients and their caregivers: a cross sectional study in a rehabilitation center Khartoum - Sudan (2014-2015). J Neurosci Rural Pract. 2016; 7(3):355-361.

10. Tugui RD, Antonescu D. Cerebral palsy gait: clinical importance. Maedica (Buchar). 2013;8(4):388-393.

11. Haak P, Lenski M, Hidecker MJ, Li M, Paneth N. Cerebral palsy and aging. Dev Med Child Neurol. 2009;51(04):16-23.

12. Hoon AH, Stashinko EE, Nagae LM, et al. Sensory and motor deficits in children with cerebral palsy born preterm correlate with diffusion tensor imaging abnormalities in thalamocortical pathways. Dev Med Child Neurol. 2009;51(9):697-704.

13. Winters T, Gage J, Hicks R. Gait patterns in spastic hemiplegia in children and young adults. J Bone Joint Surg Am. 1987;69(3):437-441.

14. Sojka AM, Stuberg WA, Knutson LM, Karst GM. Kinematic and electromyographic characteristics of children with cerebral palsy who exhibit genu recurvatum. Arch Phys Med Rehabil. 1995;76(6):558-565.

15. Abel MF, Blanco JS, Pavlovich L, Damiano DL. Asymmetric hip deformity and subluxation in cerebral palsy: an analysis of surgical treatment. J Pediatr Orthop. 1999;19(4):479-485.

16. El-Shamy SM, Abdelaal AA. WalkAide efficacy on gait and energy expenditure in children with hemiplegic cerebral palsy: a randomized controlled trial. Am J Phys Med Rehabil. 2016;95(9):629-638.

17. Galli M, Cimolin V, Rigoldi C, Tenore N, Albertini G. Gait patterns in hemiplegic children with cerebral palsy: comparison of right and left hemiplegia. Res Dev Disabil. 2010;31(6):1340-1345.

18. Bourgeois AB, Mariani B, Aminian K, Zambelli PY, Newman CJ. Spatio-temporal gait analysis in children with cerebral palsy using, foot-worn inertial sensors. Gait Posture. 2014;39(1):436-442.
19. Morgan P, Murphy A, Opheim A, McGinley J. Gait characteristics, balance performance and falls in ambulant adults with cerebral palsy: an observational study. Gait Posture. 2016;48:243-248.

20. Okoshi Y, Itoh M, Takashima S. Characteristic neuropathology and plasticity in periventricular leukomalacia. Pediatr Neurol. 2001;25(3): 221-226.

21. Cooper J, Majnemer A, Rosenblatt B, Birnbaum R. The determination of sensory deficits in children with hemiplegic cerebral palsy. $J$ Child Neurol. 1995;10(4):300-309.

22. Mackie $S$, Shaw $P$, Lenroot R, et al. Cerebellar development and clinical outcome in attention deficit hyperactivity disorder. Am J Psychiatry. 2007;164(4):647-655.

23. Reilly DS, Woollacott MH, van Donkelaar P, Saavedra S. The interaction between executive attention and postural control in dual-task conditions: children with cerebral palsy. Arch Phys Med Rehabil. 2008;89(5):834-842.

24. Courchesne E, Townsend J, Akshoomoff NA, et al. Impairment in shifting attention in autistic and cerebellar patients. Behav Neurosci. 1994;108(5):848-865.

25. Judas M, Rados M, Jovanov-Milosevic N, Hrabac P, SternPadovan R, Kostovic I. Structural, immunocytochemical, and MR imaging properties of periventricular crossroads of growing cortical pathways in preterm infants. AJNR Am J Neuroradiol. 2005;26(10): 2671-2684.

26. Hung YC, Meredith GS. Influence of dual task constraints on gait performance and bimanual coordination during walking in children with unilateral cerebral palsy. Res Dev Disabil. 2014;35(4): 755-760.

27. Ghai S, Driller M, Masters R. The influence of below-knee compression garments on knee-joint proprioception. Gait Posture. Epub 2016 Aug 9 .

28. Masters RS. Knowledge, knerves and know-how: the role of explicit versus implicit knowledge in the breakdown of a complex motor skill under pressure. Br J Psychol. 1992;83(3):343-358.

29. Masters RS, Maxwell J. The theory of reinvestment. Int Rev Sport Exerc Psychol. 2008;1(2):160-183.

30. Ghai S, Ghai I, Effenberg AO. Effects of dual tasks and dual-task training on postural stability: a systematic review and meta-analysis. Clin Interv Aging. 2017;12:557-577.

31. Snider L, Majnemer A, Darsaklis V. Virtual reality as a therapeutic modality for children with cerebral palsy. Dev Neurorehabil. 2010; 13(2):120-128.

32. MacIntosh A, Vignais N, Biddiss E. Biofeedback interventions for people with cerebral palsy: a systematic review protocol. Syst Rev. 2017;6(1):3.

33. Martin L, Baker R, Harvey A. A systematic review of common physiotherapy interventions in school-aged children with cerebral palsy. Phys Occup Ther Pediatr. 2010;30(4):294-312.

34. Verschuren O, Ketelaar M, Takken T, Helders PJ, Gorter JW. Exercise programs for children with cerebral palsy: a systematic review of the literature. Am J Phys Med Rehabil. 2008;87(5):404-417.

35. Willoughby KL, Dodd KJ, Shields N. A systematic review of the effectiveness of treadmill training for children with cerebral palsy. Disabil Rehabil. 2009;31(24):1971-1979.

36. Figueiredo EM, Ferreira GB, Moreira RC, Kirkwood RN, Fetters L. Efficacy of ankle-foot orthoses on gait of children with cerebral palsy: systematic review of literature. Pediatr Phys Ther. 2008;20(3): 207-223.

37. Novak I, McIntyre S, Morgan C, et al. A systematic review of interventions for children with cerebral palsy: state of the evidence. Dev Med Child Neurol. 2013;55(10):885-910.

38. Shin YK, Chong HJ, Kim SJ, Cho SR. Effect of rhythmic auditory stimulation on hemiplegic gait patterns. Yonsei Med J. 2015;56(6): 1703-1713.

39. Kim SJ, Kwak EE, Park ES, Cho SR. Differential effects of rhythmic auditory stimulation and neurodevelopmental treatment/Bobath on gait patterns in adults with cerebral palsy: a randomized controlled trial. Clin Rehabil. 2012;26(10):904-914. 
40. Kim SJ, Shin YK, Yoo GE, Chong HJ, Cho SR. Changes in gait patterns induced by rhythmic auditory stimulation for adolescents with acquired brain injury. Ann N Y Acad Sci. 2016;1385(1):53-62.

41. Baram Y, Lenger R. Gait improvement in patients with cerebral palsy by visual and auditory feedback. Neuromodulation. 2012;15(1):48-52.

42. Fujioka T, Trainor LJ, Large EW, Ross B. Internalized timing of isochronous sounds is represented in neuromagnetic $\beta$ oscillations. J Neurosci. 2012;32(5):1791-1802.

43. Effenberg AO, Fehse U, Schmitz G, Krueger B, Mechling H. Movement sonification: effects on motor learning beyond rhythmic adjustments. Front Neurosci. 2016;10:219.

44. Schmitz G, Mohammadi B, Hammer A, et al. Observation of sonified movements engages a basal ganglia frontocortical network. $B M C$ Neurosci. 2013; 14:32.

45. Heremans E, Nieuwboer A, Spildooren J, et al. Cued motor imagery in patients with multiple sclerosis. Neuroscience. 2012;206:115-121.

46. Heremans E, Nieuwboer A, Feys P, et al. External cueing improves motor imagery quality in patients with Parkinson disease. Neurorehabil Neural Repair. 2012;26(1):27-35.

47. Miller RA, Thaut MH, McIntosh GC, Rice RR. Components of EMG symmetry and variability in parkinsonian and healthy elderly gait. Electroencephalogr Clin Neurophysiol. 1996;101(1):1-7.

48. Luft AR, McCombe-Waller S, Whitall J, et al. Repetitive bilateral arm training and motor cortex activation in chronic stroke: a randomized controlled trial. JAMA. 2004;292(15):1853-1861.

49. Rochester L, Baker K, Nieuwboer A, Burn D. Targeting dopa-sensitive and dopa-resistant gait dysfunction in Parkinson's disease: selective responses to internal and external cues. Mov Dis. 2011;26(3):430-435.

50. Zhao Y, Nonnekes J, Storcken EJ, et al. Feasibility of external rhythmic cueing with the Google Glass for improving gait in people with Parkinson's disease. J Neurol. 2016;263(6):1156-1165.

51. Rodger MW, Craig CM. Beyond the metronome: auditory events and music may afford more than just interval durations as gait cues in Parkinson's disease. Front Neurosci. 2016;10:272.

52. Wu YW, Xing G, Fuentes-Afflick E, Danielson B, Smith LH, Gilbert WM. Racial, ethnic, and socioeconomic disparities in the prevalence of cerebral palsy. Pediatrics. 2011;127(3):e674-e681.

53. Oskoui M, Messerlian C, Blair A, Gamache P, Shevell M. Variation in cerebral palsy profile by socio-economic status. Dev Med Child Neurol. 2016;58(2):160-166.

54. Rocha PA, Porfírio GM, Ferraz HB, Trevisani VF. Effects of external cues on gait parameters of Parkinson's disease patients: a systematic review. Clin Neurol Neurosurg. 2014;124:127-134.

55. Lim I, van Wegen E, de Goede C, et al. Effects of external rhythmical cueing on gait in patients with Parkinson's disease: a systematic review. Clin Rehabil. 2005;19(7):695-713.

56. Spaulding SJ, Barber B, Colby M, Cormack B, Mick T, Jenkins ME. Cueing and gait improvement among people with Parkinson's disease: a meta-analysis. Arch Phys Med Rehabil. 2013;94(3): 562-570

57. Nascimento LR, de Oliveira CQ, Ada L, Michaelsen SM, TeixeiraSalmela LF. Walking training with cueing of cadence improves walking speed and stride length after stroke more than walking training alone: a systematic review. J Physiother. 2015;61(1):10-15.

58. Yoo GE, Kim SJ. Rhythmic auditory cueing in motor rehabilitation for stroke patients: systematic review and meta-analysis. $J$ Music Ther. 2016;53(2):149-177.

59. Zhang Y, Cai J, Zhang Y, Ren T, Zhao M, Zhao Q. Improvement in stroke-induced motor dysfunction by music-supported therapy: a systematic review and meta-analysis. Sci Rep. 2016;6:38521.

60. Liberati A, Altman DG, Tetzlaff J, et al. The PRISMA statement for reporting systematic reviews and meta-analyses of studies that evaluate health care interventions: explanation and elaboration. Ann Intern Med. 2009;151(4):W65-W94

61. Wiart L, Kolaski K, Butler C, et al. Interrater reliability and convergent validity of the American Academy for Cerebral Palsy and Developmental Medicine methodology for conducting systematic reviews. Dev Med Child Neurol. 2012;54(7):606-611.
62. de Morton NA. The PEDro scale is a valid measure of the methodological quality of clinical trials: a demographic study. Aust J Physiother. 2009;55(2):129-133.

63. Maher CG, Sherrington C, Herbert RD, Moseley AM, Elkins M. Reliability of the PEDro scale for rating quality of randomized controlled trials. Phys Ther. 2003;83(8):713-721.

64. Teasell R. EBRSR: evidence-based review of stroke rehabilitation. 2008. Available from: www.ebrsr.com. Accessed November 2, 2017.

65. Ramsey L, Winder RJ, McVeigh JG. The effectiveness of working wrist splints in adults with rheumatoid arthritis: a mixed methods systematic review. J Rehabil Med. 2014;46(6):481-492.

66. Borenstein M, Hedges LV, Higgins J, Rothstein HR. A basic introduction to fixed-effect and random-effects models for meta-analysis. Res Synth Methods. 2010;1(2):97-111.

67. Higgins JP, Green S. Cochrane Handbook for Systematic Reviews of Interventions. Hoboken (NJ): John Wiley \& Sons; 2011.

68. Cumming G. Understanding the New Statistics: Effect Sizes, Confidence Intervals, and Meta-analysis. New York: Routledge; 2013.

69. Cohen J. Statistical Power Analysis for the Behavioral Sciences. 2nd ed. Hillsdale (NJ): Lawrence Erlbaum Associates; 1988.

70. Efraimidou V, Tsimaras V, Proios M, et al. The effect of a music and movement program on gait, balance and psychological parameters of adults with cerebral palsy. J Phys Educ Sport. 2016;16(4): 1357-1364.

71. Bolier L, Haverman M, Westerhof GJ, Riper H, Smit F, Bohlmeijer E. Positive psychology interventions: a meta-analysis of randomized controlled studies. BMC Public Health. 2013;13:119.

72. Kim JS, Oh DW. Home-based auditory stimulation training for gait rehabilitation of chronic stroke patients. J Phys Ther Sci. 2012;24(8): 775-777.

73. Wang TH, Peng YC, Chen YL, et al. A home-based program using patterned sensory enhancement improves resistance exercise effects for children with cerebral palsy: a randomized controlled trial. Neurorehabil Neural Repair. 2013;27(8):684-694.

74. Kwak EE. Effect of rhythmic auditory stimulation on gait performance in children with spastic cerebral palsy. J Music Ther. 2007;44(3): $198-216$.

75. Jiang A. The Effect of Rhythmic Auditory Stimulation on Gait in Young Children with Spastic Cerebral Palsy [master's thesis]. Miami: University of Miami; 2013.

76. Kim SJ, Kwak EE, Park ES, et al. Changes in gait patterns with rhythmic auditory stimulation in adults with cerebral palsy. Neurorehabilitation. 2011;29(3):233-241.

77. Varsamis P, Staikopoulos K, Kartasidou L. Effect of rhythmic auditory stimulation on controlling stepping cadence of individuals with mental retardation and cerebral palsy. Int J Spec Educ. 2012;27(3):68-75.

78. Inder TE, Warfield SK, Wang H, Huppi PS, Volpe JJ. Abnormal cerebral structure is present at term in premature infants. Pediatrics. 2005; 115(2):286-294

79. Messerschmidt A, Fuiko R, Prayer D, et al. Disrupted cerebellar development in preterm infants is associated with impaired neurodevelopmental outcome. Eur J Pediatr. 2008;167(10):1141-1147.

80. Dyet LE, Kennea N, Counsell SJ, et al. Natural history of brain lesions in extremely preterm infants studied with serial magnetic resonance imaging from birth and neurodevelopmental assessment. Pediatrics. 2006;118(2):536-548.

81. Pierson CR, Folkerth RD, Billiards SS, et al. Gray matter injury associated with periventricular leukomalacia in the premature infant. Acta Neuropathol. 2007;114(6):619-631.

82. Callisaya ML, Beare R, Phan TG, et al. Brain structural change and gait decline: a longitudinal population-based study. J Am Geriatr Soc. 2013;61(7):1074-1079.

83. Grasso R, Peppe A, Stratta F, et al. Basal ganglia and gait control: apomorphine administration and internal pallidum stimulation in Parkinson's disease. Exp Brain Res. 1999;126(2):139-148.

84. Raz N, Rodrigue KM, Kennedy KM, Head D, Gunning-Dixon F, Acker JD. Differential aging of the human striatum: longitudinal evidence. AJNR Am J Neuroradiol. 2003;24(9):1849-1856. 
85. Wolpe N, Ingram JN, Tsvetanov KA, et al. Ageing increases reliance on sensorimotor prediction through structural and functional differences in frontostriatal circuits. Nat Commun. 2016;7:13034.

86. Seidler RD, Bernard JA, Burutolu TB, et al. Motor control and aging: links to age-related brain structural, functional, and biochemical effects. Neurosci Biobehav Rev. 2010;34(5):721-733.

87. Nombela C, Hughes LE, Owen AM, Grahn JA. Into the groove: can rhythm influence Parkinson's disease? Neurosci Biobehav Rev. 2013;37(10):2564-2570.

88. Aboutorabi A, Arazpour M, Bahramizadeh M, Hutchins SW, Fadayevatan R. The effect of aging on gait parameters in able-bodied older subjects: a literature review. Aging Clin Exp Res. 2016;28(3): 393-405.

89. Perry MC, Carville SF, Smith IC, Rutherford OM, Newham DJ. Strength, power output and symmetry of leg muscles: effect of age and history of falling. Eur J Appl Physiol. 2007;100(5):553-561.

90. Schmidt RA. Frequent augmented feedback can degrade learning: evidence and interpretations. In: Requin R, Stelmach GE, editors. Tutorials in Motor Neurosci. Springer; 1991:59-75.

91. Winstein CJ, Pohl PS, Lewthwaite R. Effects of physical guidance and knowledge of results on motor learning: support for the guidance hypothesis. Res $Q$ Exerc Sport. 1994;65(4):316-323.

92. Hameed S, Ferris T, Jayaraman S, Sarter N. Using informative peripheral visual and tactile cues to support task and interruption management. Hum Factors. 2009;51(2):126-135.

93. Elsinger CL, Rao SM, Zimbelman JL, Reynolds NC, Blindauer KA, Hoffmann RG. Neural basis for impaired time reproduction in Parkinson's disease: an fMRI study. J Int Neuropsychol Soc. 2003; 9(7):1088-1098.

94. Hausdorff JM, Lowenthal J, Herman T, Gruendlinger L, Peretz C, Giladi N. Rhythmic auditory stimulation modulates gait variability in Parkinson's disease. Eur J Neurosci. 2007;26(8):2369-2375.

95. Rubinstein TC, Giladi N, Hausdorff JM. The power of cueing to circumvent dopamine deficits: a review of physical therapy treatment of gait disturbances in Parkinson's disease. Mov Disord. 2002;17(6):1148-1160.

96. Cunnington R, Iansek R, Bradshaw JL, Phillips JG. Movement-related potentials in Parkinson's disease. Brain. 1995;118(4):935-950.

97. Cabeza R, Anderson ND, Locantore JK, McIntosh AR. Aging gracefully: compensatory brain activity in high-performing older adults. Neuroimage. 2002;17(3):1394-1402.

98. Tierney A, Kraus N. The ability to move to a beat is linked to the consistency of neural responses to sound. J Neurosci. 2013;33(38): 14981-14988.

99. Debaere F, Wenderoth N, Sunaert S, Van Hecke P, Swinnen SP. Internal vs external generation of movements: differential neural pathways involved in bimanual coordination performed in the presence or absence of augmented visual feedback. Neuroimage. 2003;19(3):764-776.

100. Asanuma H, Keller A. Neuronal mechanisms of motor learning in mammals. Neuroreport. 1991;2(5):217-224.

101. Suh JH, Han SJ, Jeon SY, et al. Effect of rhythmic auditory stimulation on gait and balance in hemiplegic stroke patients. Neurorehabilitation. 2014;34(1):193-199.

102. Thaut MH, McIntosh GC, Rice RR, Miller RA, Rathbun J, Brault JM. Rhythmic auditory stimulation in gait training for Parkinson's disease patients. Mov Disord. 1996;11(2):193-200.

103. Thaut MH. Rhythm, Music, and the Brain: Scientific Foundations and Clinical Applications. New York: Routledge; 2005.

104. Lohnes CA, Earhart GM. The impact of attentional, auditory, and combined cues on walking during single and cognitive dual tasks in Parkinson disease. Gait Posture. 2011;33(3):478-483.

105. Young WR, Rodger MW, Craig CM. Auditory observation of stepping actions can cue both spatial and temporal components of gait in Parkinson's disease patients. Neuropsychologia. 2014;57: 140-153.
106. Dotov D, Bayard S, de Cock VC, et al. Biologically-variable rhythmic auditory cues are superior to isochronous cues in fostering natural gait variability in Parkinson's disease. Gait Posture. 2017;51:64-69.

107. Gaver WW. How do we hear in the world? Explorations in ecological acoustics. Ecol Psychol. 1993;5(4):285-313.

108. Rizzo JR, Raghavan P, McCrery JR, Oh-Park M, Verghese J. Effects of emotionally charged auditory stimulation on gait performance in the elderly: a preliminary study. Arch Phys Med Rehabil. 2015; 96(4):690-696.

109. Franěk M, van Noorden L, Režný L. Tempo and walking speed with music in the urban context. Front Psychol. 2014;5:1361.

110. Leman M, Moelants D, Varewyck M, Styns F, van Noorden L, Martens JP. Activating and relaxing music entrains the speed of beat synchronized walking. PLoS One. 2013;8(7):e67932.

111. Fang R, Ye S, Huangfu J, Calimag DP. Music therapy is a potential intervention for cognition of Alzheimer's disease: a mini-review. Transl Neurodegener. 2017;6:2.

112. Stork MJ, Kwan MY, Gibala MJ, Ginis KA. Music enhances performance and perceived enjoyment of sprint interval exercise. Med Sci Sports Exerc. 2015;47(5):1052-1060.

113. Rochester L, Rafferty D, Dotchin C, Msuya O, Minde V, Walker R. The effect of cueing therapy on single and dual-task gait in a drug naïve population of people with Parkinson's disease in northern Tanzania. Mov Disord. 2010;25(7):906-911.

114. Godara B, Nikita KS. Wireless Mobile Communication and Healthcare: Third International Conference, MobiHealth 2012, Paris, France, November 21-23, 2012 - Revised Selected Papers. Heidelberg: Springer; 2013.

115. Hove MJ, Suzuki K, Uchitomi H, Orimo S, Miyake Y. Interactive rhythmic auditory stimulation reinstates natural 1/f timing in gait of Parkinson's patients. PLoS One. 2012;7(3):e32600.

116. Lopez WO, Higuera CA, Fonoff ET, Souza CO, Albicker U, Martinez JA. ListenMee and ListenMee smartphone application: synchronizing walking to rhythmic auditory cues to improve gait in Parkinson's disease. Hum Mov Sci. 2014;37:147-156.

117. Poushter J. Smartphone ownership and Internet usage continues to climb in emerging economies. 2016. Available from: http:// www.pewglobal.org/2016/02/22/smartphone-ownership-andinternet-usage-continues-to-climb-in-emerging-economies. Accessed November 2, 2017.

118. Fragala-Pinkham MA, Smith HJ, Lombard KA, Barlow C, O’Neil ME. Aquatic aerobic exercise for children with cerebral palsy: a pilot intervention study. Physiother Theory Pract. 2014;30(2):69-78.

119. Combs SA, Dugan EL, Passmore M, et al. Balance, balance confidence, and health-related quality of life in persons with chronic stroke after body weight-supported treadmill training. Arch Phys Med Rehabil. 2010;91(12):1914-1919.

120. Kim D. Correlation between physical function, cognitive function, and health-related quality of life in elderly persons. J Phys Ther Sci. 2016;28(6):1844-1848.

121. Ghai S, Ghai I, Effenberg AO. Effect of rhythmic auditory cueing on aging gait: a systematic review and meta-analysis. $A \& D$. In press 2017.

122. Ghai S, Ghai I, Schmitz G, Effenberg AO. Effect of rhythmic auditory cueing on parkinsonian gait: a systematic review and meta-analysis. Sci rep. In press 2017.

123. Ghai S, Schmitz G, Hwang TH, Effenberg AO. Effects of real-time auditory feedback on proprioceptive accuracy. In: the 22nd Annual Congress of European College of Sports Science, Essen, Germany, July 5-8, 2017; MetropolisRuhr - Germany.

124. Ghai S, Driller M, Ghai I. Effects of joint stabilizers on proprioception and stability: a systematic review and meta-analysis. Phys Ther Sport. 2017;25:65-75.

125. Ghai S. Proprioception and performance: the role of below-knee compression garments and secondary tasks [dissertation]. Hamilton: University of Waikato; 2016. 


\section{Supplementary materials}

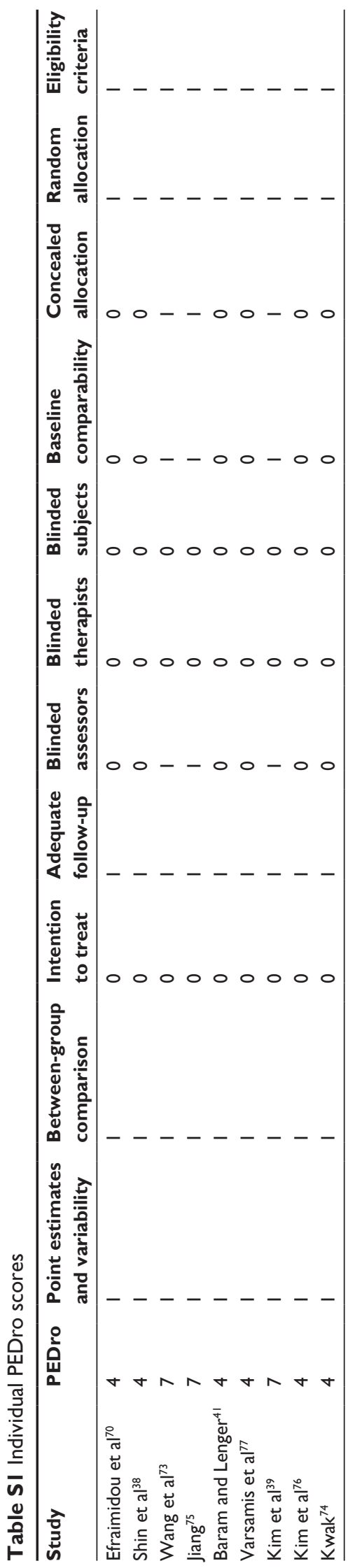

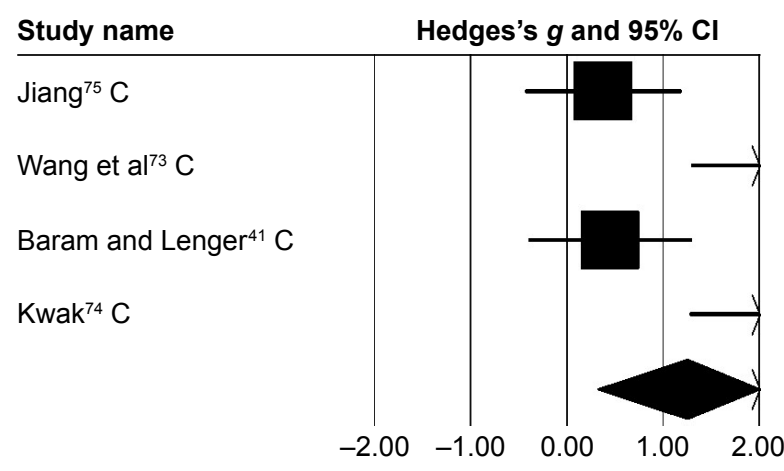

Figure SI Forest plot illustrating individual studies evaluating the effects of rhythmic auditory cueing on gait velocity in children with cerebral palsy.

Notes: Negative effects indicate reduction in gait velocity, positive effects enhancement in gait velocity. Weighted-effect sizes - Hedge's $g$ (boxes) and $95 \% \mathrm{Cl}$ (whiskers) - demonstrate repositioning errors for individual studies. The diamond represents pooled effect sizes and $95 \% \mathrm{Cl}$. Negative mean differences indicate favorable outcomes for control groups, positive mean differences favorable outcomes for experimental groups.

Abbreviation: $C$, children.

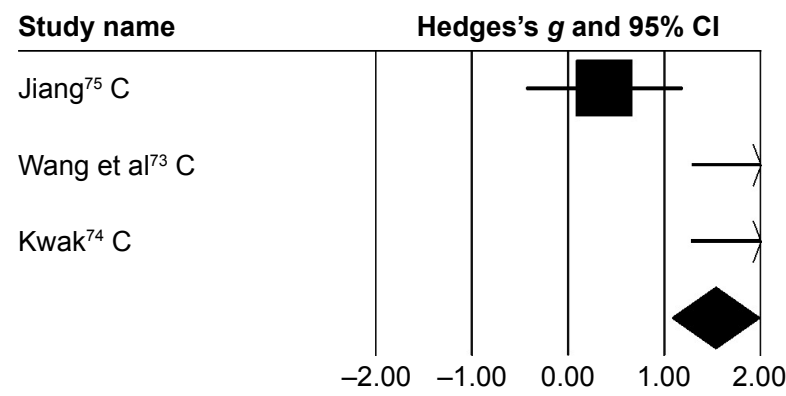

Figure S2 Forest plot illustrating individual studies evaluating the effects of rhythmic auditory cueing on gait velocity in children with cerebral palsy posttraining.

Notes: Negative effects indicate reduction in gait velocity, positive effect sizes enhancement in gait velocity. Weighted-effect sizes - Hedge's $g$ (boxes) and $95 \% \mathrm{Cl}$ (whiskers) - demonstrate repositioning errors for individual studies. The diamond represents pooled effect sizes and $95 \% \mathrm{Cl}$. Negative mean differences indicate favorable outcomes for control groups, positive mean differences favorable outcomes for experimental groups.

Abbreviation: $C$, children.

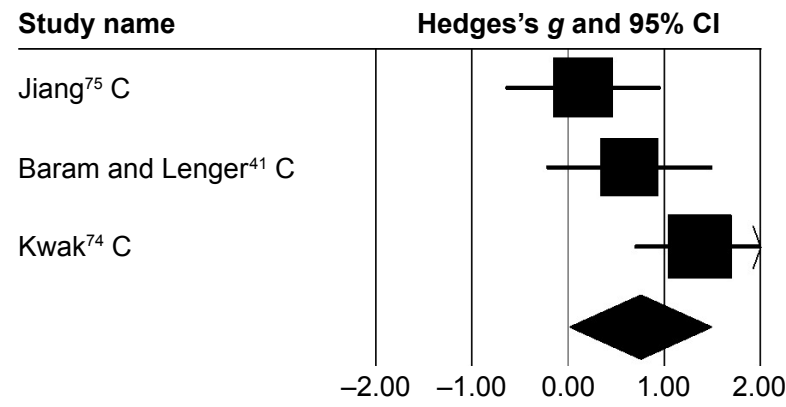

Figure S3 Forest plot illustrating individual studies evaluating the effects of rhythmic auditory cueing on stride length in children with cerebral palsy.

Notes: Negative effects indicate reduction in stride length, positive effects enhancement in stride length. Weighted-effect sizes - Hedge's $g$ (boxes) and $95 \% \mathrm{Cl}$ (whiskers) - demonstrate repositioning errors for individual studies. The diamond represents pooled effect sizes and $95 \% \mathrm{Cl}$. Negative mean difference indicate favorable outcomes for control groups, positive mean difference favorable outcomes for experimental groups.

Abbreviation: $\mathrm{C}$, children. 


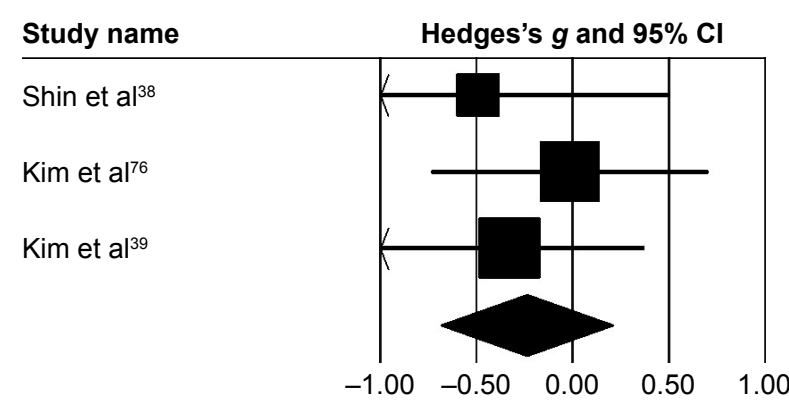

Figure S4 Forest plot illustrating individual studies evaluating the effects of rhythmic auditory cueing on pelvic kinematics in adults with cerebral palsy.

Notes: Negative effects indicate reduction in pelvic kinematics, positive effects enhancement in pelvic kinematics. Weighted-effect sizes - Hedge's g (boxes) and $95 \% \mathrm{Cl}$ (whiskers) - demonstrate repositioning errors for individual studies. The diamond represents pooled effect sizes and $95 \% \mathrm{Cl}$. Negative mean differences indicates favorable outcomes for control groups, positive mean differences favorable outcomes for experimental groups.

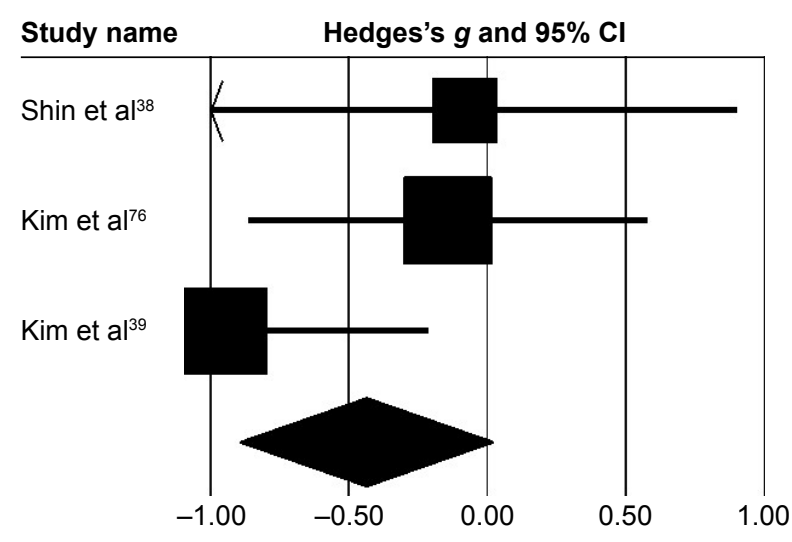

Figure S5 Forest plot illustrating individual studies evaluating the effects of rhythmic auditory cueing on hip kinematics in adults with cerebral palsy.

Notes: Negative effects indicate reduction in hip kinematics, positive effects enhancement in hip kinematics. Weighted-effect sizes - Hedge's $g$ (boxes) and $95 \% \mathrm{Cl}$ (whiskers) - demonstrate repositioning errors for individual studies. The diamond represents pooled effect sizes and $95 \% \mathrm{Cl}$. Negative mean differences indicate favorable outcomes for control groups, positive mean differences favorable outcomes for experimental groups.

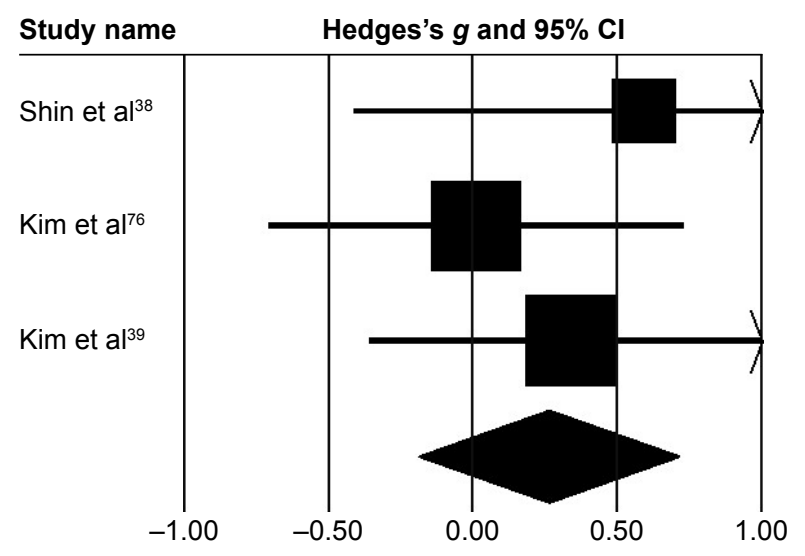

Figure S6 Forest plot illustrating individual studies evaluating the effects of rhythmic auditory cueing on knee kinematics in adults with cerebral palsy.

Notes: Negative effects indicate reduction in knee kinematics, positive effects enhancement in knee kinematics. Weighted-effect sizes - Hedge's g (boxes) and 95\% Cl (whiskers) - demonstrate repositioning errors for individual studies. The diamond represents pooled effect sizes and $95 \% \mathrm{Cl}$. Negative mean differences indicate favorable outcomes for control groups, positive mean differences favorable outcomes for experimental groups.

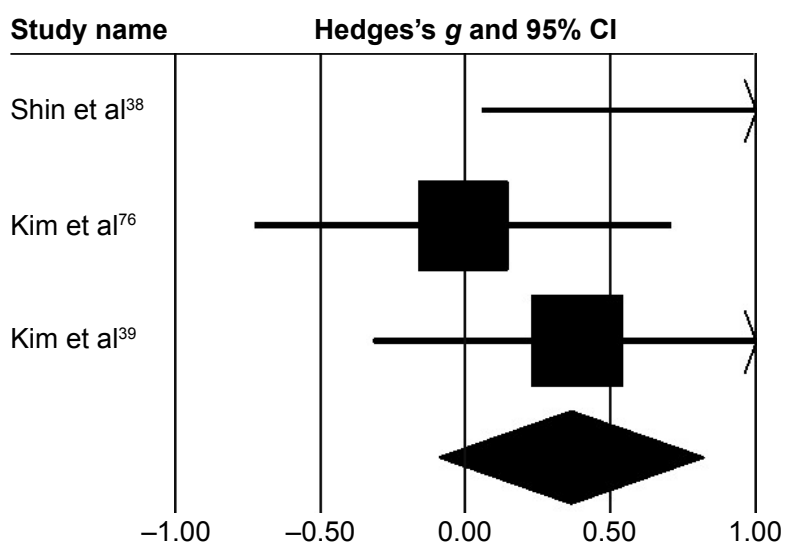

Figure S7 Forest plot illustrating individual studies evaluating the effects of rhythmic auditory cueing on ankle kinematics in adults with cerebral palsy.

Notes: Negative effect sizes indicate reduction in ankle kinematics, positive effects enhancement in ankle kinematics. Weighted-effect sizes - Hedge's $g$ (boxes) and $95 \% \mathrm{Cl}$ (whiskers) - demonstrating repositioning errors for individual studies. The diamond represents pooled effect sizes and $95 \% \mathrm{Cl}$. Negative mean differences indicate favorable outcomes for control groups, positive mean difference favorable outcomes for experimental groups.

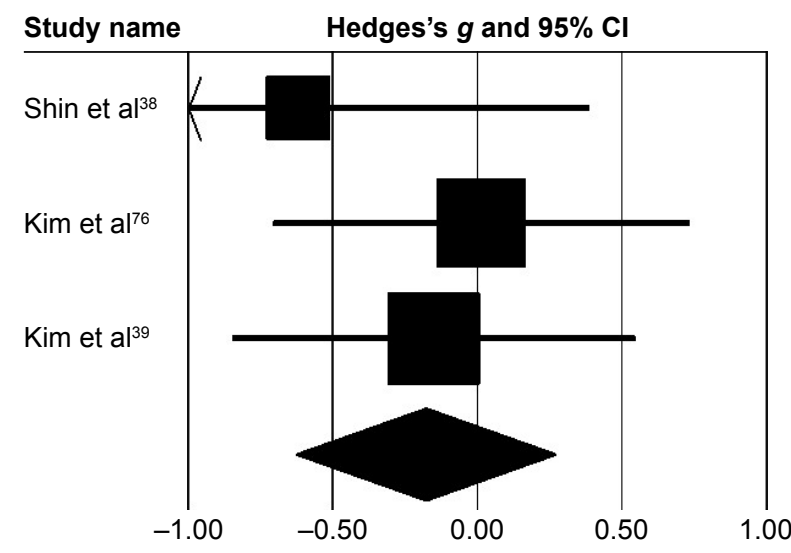

Figure S8 Forest plot illustrating individual studies evaluating the effects of rhythmic auditory cueing on foot kinematics in adults with cerebral palsy.

Notes: Negative effects indicated reduction in foot kinematics, positive effects enhancement in foot kinematics. Weighted-effect sizes - Hedge's $g$ (boxes) and $95 \% \mathrm{Cl}$ (whiskers) - demonstrate repositioning errors for individual studies. The diamond represents pooled effect sizes and $95 \% \mathrm{Cl}$. Negative mean differences indicate favorable outcomes for control groups, positive mean differences favorable outcomes for experimental groups. 
Neuropsychiatric Disease and Treatment

Dovepress

\section{Publish your work in this journal}

Neuropsychiatric Disease and Treatment is an international, peerreviewed journal of clinical therapeutics and pharmacology focusing on concise rapid reporting of clinical or pre-clinical studies on a range of neuropsychiatric and neurological disorders. This journal is indexed on PubMed Central, the 'PsycINFO' database and CAS,

and is the official journal of The International Neuropsychiatric Association (INA). The manuscript management system is completely online and includes a very quick and fair peer-review system, which is all easy to use. Visit http://www.dovepress.com/testimonials.php to read real quotes from published authors.

Submit your manuscript here: http://www.dovepress.com/neuropsychiatric-disease-and-treatment-journal 